\title{
Wet Spinning of Silk Fibroin-based Core-sheath Fibers
}

Pui Fai NG, ${ }^{1}$ Ka I LEE, ${ }^{1}$ Shengfei MENG, ${ }^{2}$ Jidong ZHANG, ${ }^{2}$ Yuhong WANG, ${ }^{3}$ Bin FEI $I^{1 *}$

1, Institute of Textiles \& Clothing, Hong Kong Polytechnic University, 11 Yuk Choi Road, Kowloon, Hong Kong, China.

2, State Key Laboratory of Polymer Physics and Chemistry, Changchun Institute of Applied Chemistry, Chinese Academy of Sciences, No. 5625, Ren Min Street, Changchun, China

3, Department of Civil and Environmental Engineering, Hong Kong Polytechnic University, 11 Yuk Choi Road, Kowloon, Hong Kong, China

Corresponding author. E-mail: tcfeibepolyu.edu.hk. Tel: +85227664795. 


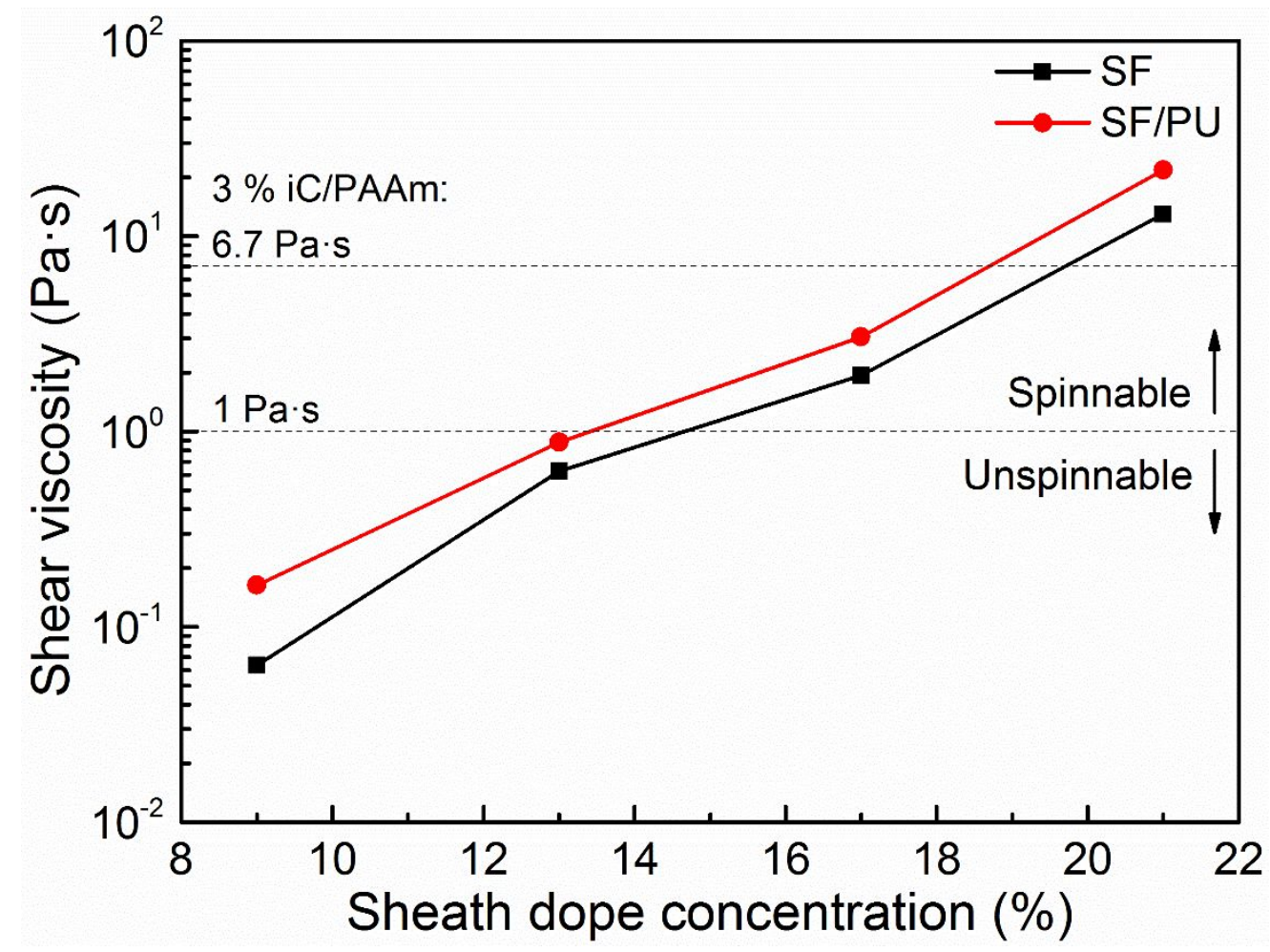

Figure S1. Relationship between polymer concentration and shear viscosity of SF and SF/PU sheath dopes at a shear rate of $10 \mathrm{~s}^{-1}$.
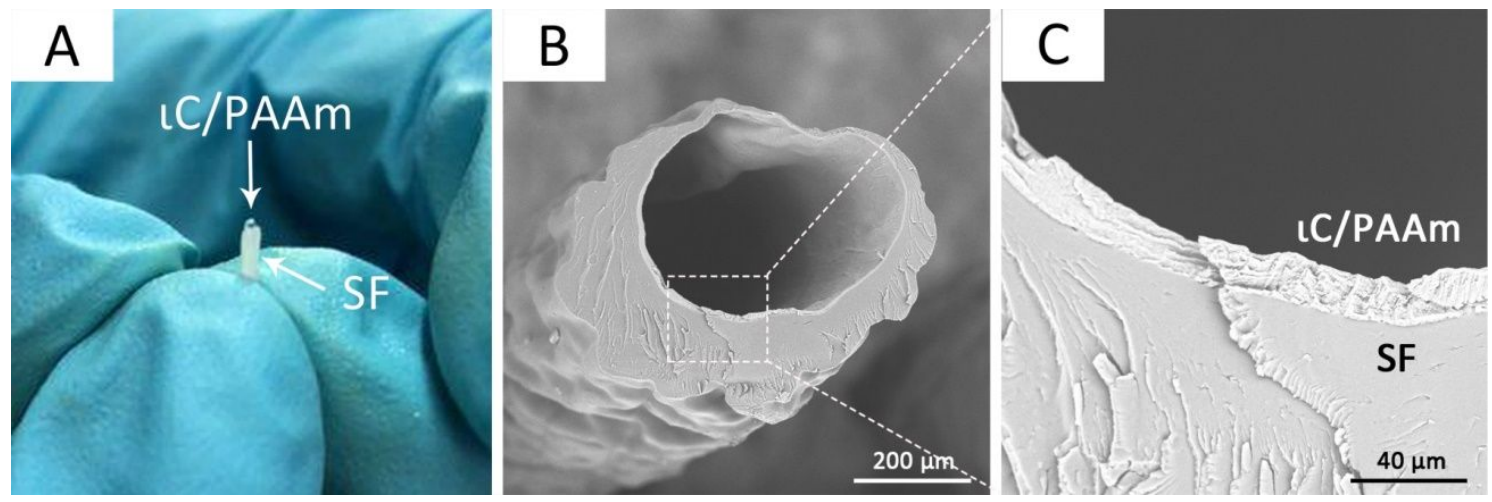

Figure S2. The core-sheath fiber spun from iC/PAAm core dope and $17 \%$ SF sheath dope: fiber end observed by naked eyes (A) and SEM (B and C). 

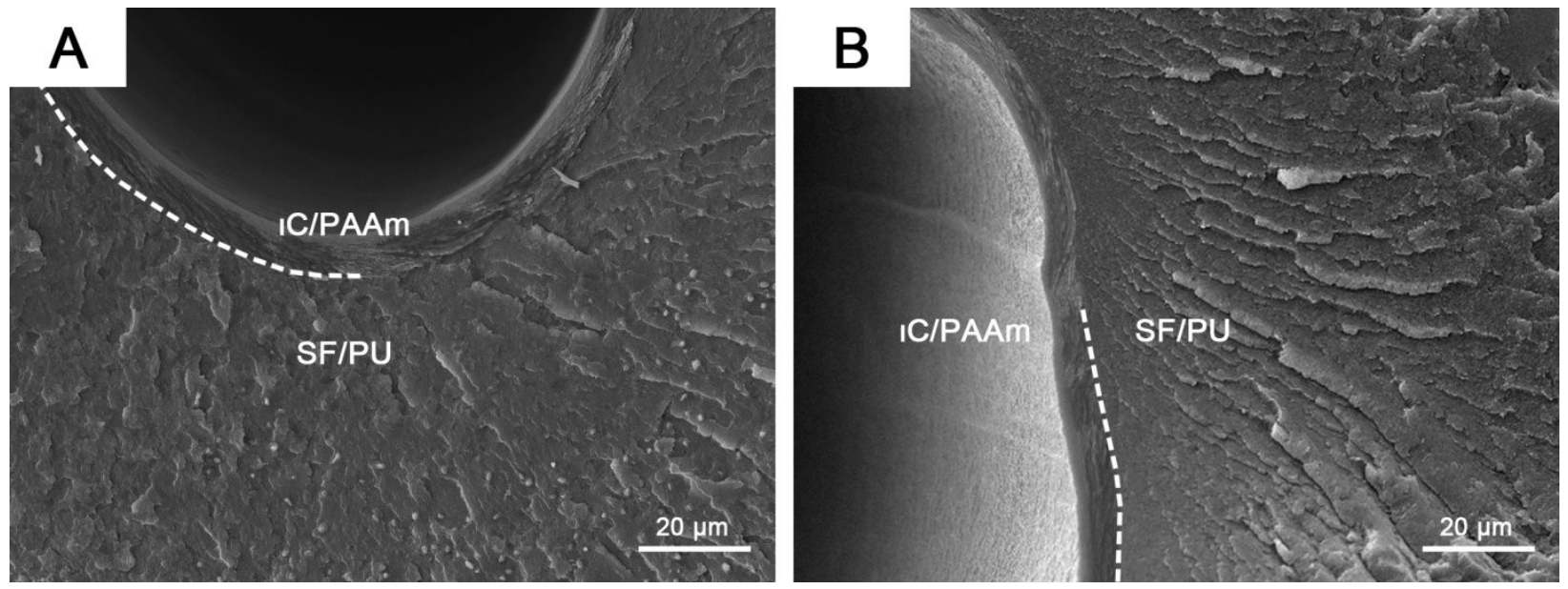

Figure S3. The cross-section of as-spun fiber from iC/PAAm core dope and $17 \% \mathrm{SF} / \mathrm{PU}$ sheath dope: (A) unrinsed, (B) rinsed. 

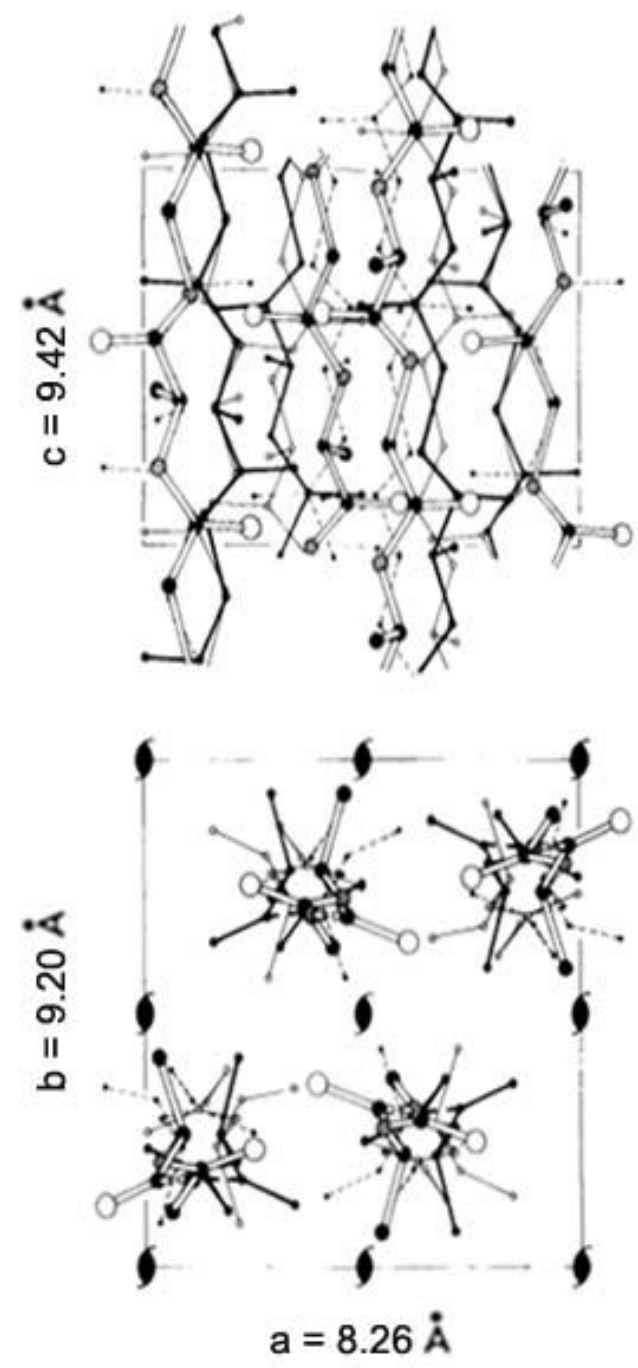

Figure S4. The illustration of fibroin $\beta$-sheet crystal unit derived from the 2D XRD measurement (the exact chain arrangement in unit cell is still subject to adjustment by further theoretical simulation $)^{56}$. 


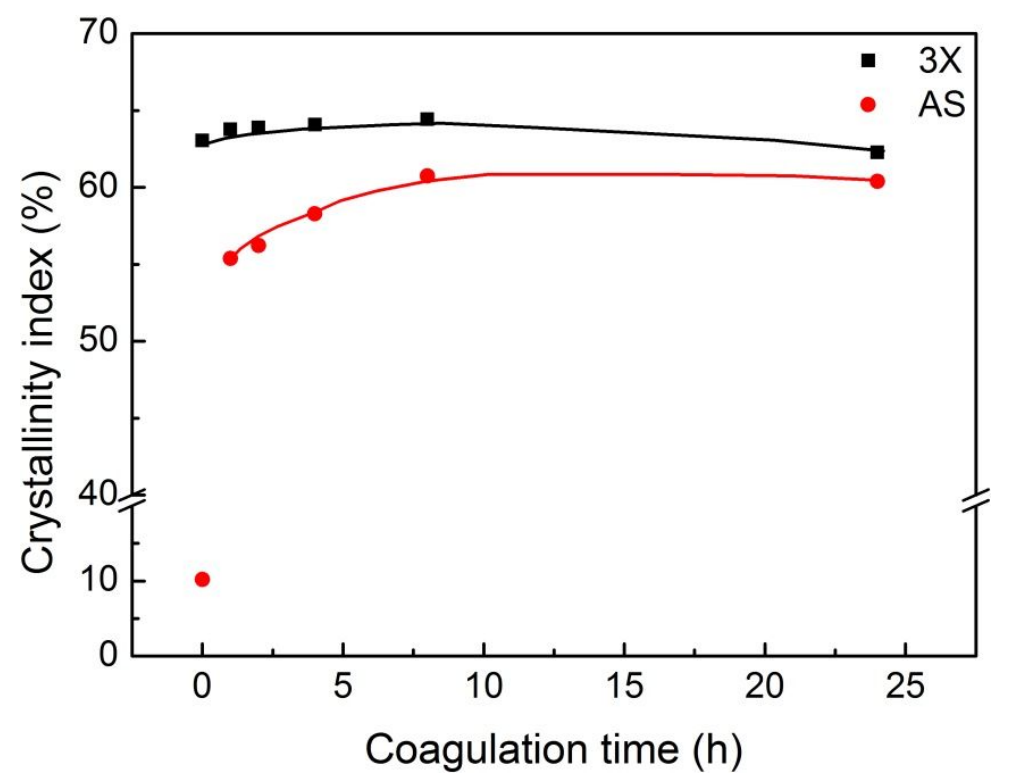

Figure S5. Effect of the coagulation time on the crystallinity index of the as-spun (AS) and postdrawn $(3.0 \mathrm{X})$ fibres derived from the peak intensity ratio at 1262 to $1230 \mathrm{~cm}^{-1}$ of their FTIR spectra.
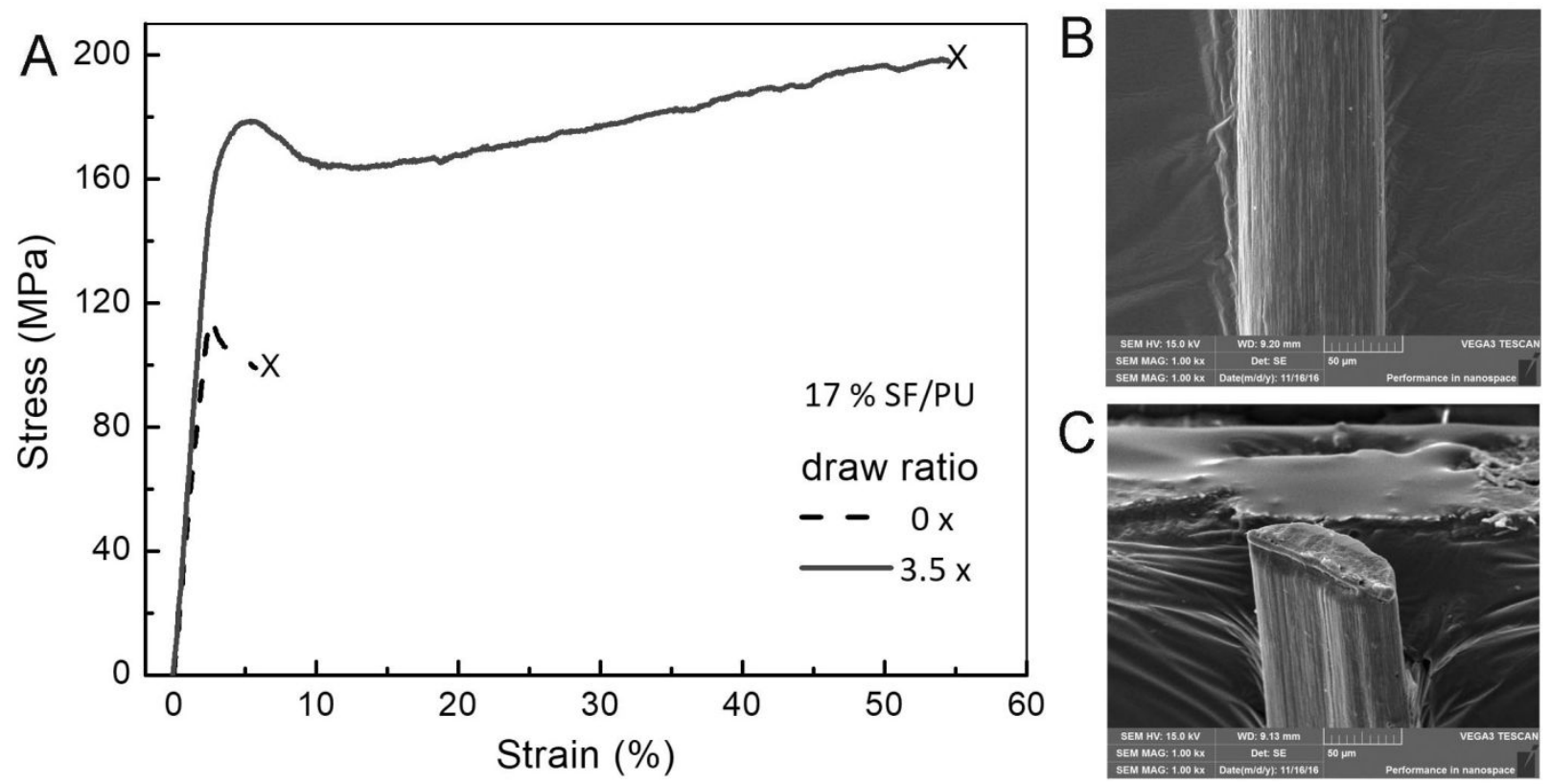

Figure S6. Tensile curves of solid fibers from $17 \% \mathrm{SF} / \mathrm{PU}$ (A); Side view and cross-section of the $3.5 \mathrm{x}$ solid fiber spun from $17 \% \mathrm{SF} / \mathrm{PU}$ (B \& C) (single 14-gauge needle, with other conditions same to that of $\mathrm{iC} / \mathrm{PAAm}-\mathrm{SF} / \mathrm{PU}$ fiber spinning). 


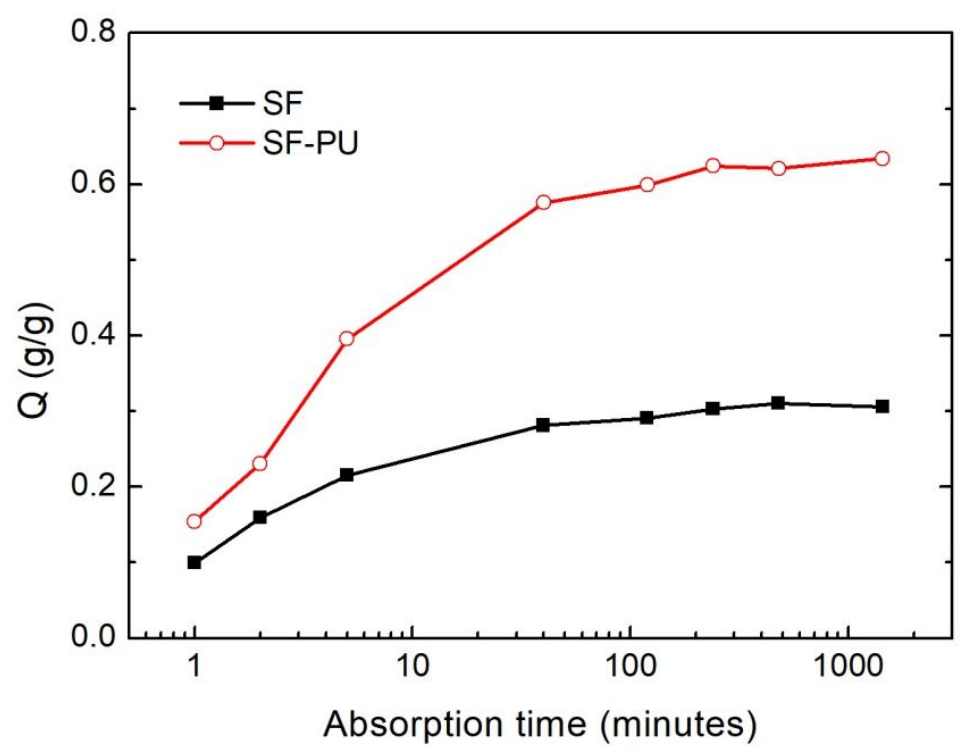

Figure s7. Water absorption dynamics of the degummed silk (SF) and as-spun solid fiber from the 17\% SF/PU dope (SF-PU, single 14gauge needle).
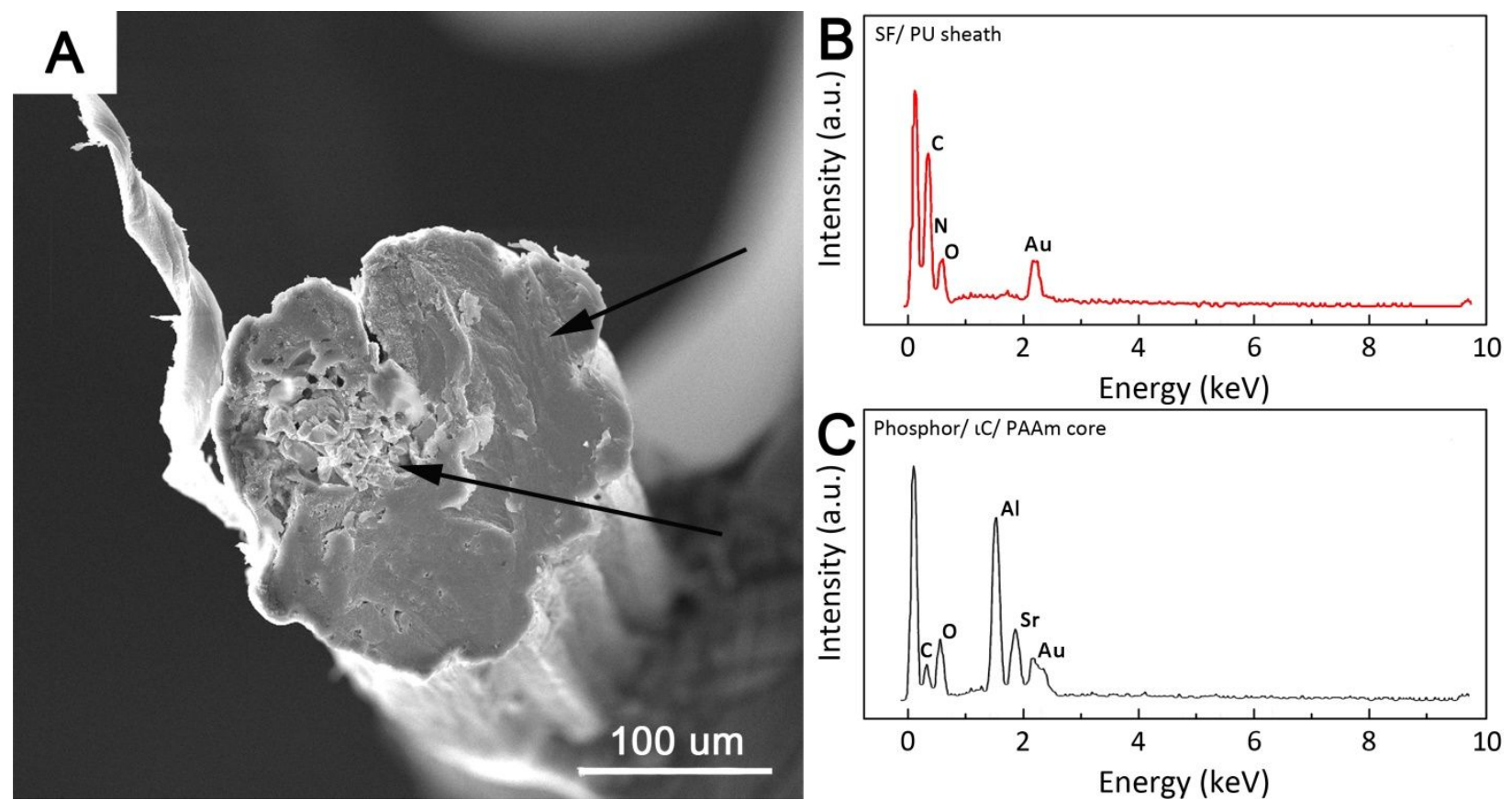

Figure S8. The rinsed as-spun fiber from pigment/iC/PAAm core dope and $17 \%$ SF/PU sheath dope: its cross-section (A) and EDS results for its sheath area (B) and core area (C). 